\title{
Updated Sniffin' Sticks normative data based on an extended sample of 9139 subjects
}

\author{
A. Oleszkiewicz ${ }^{1,2} \cdot$ V. A. Schriever ${ }^{3} \cdot$ I. $_{\text {Croy }}^{4} \cdot$ A. Hähner ${ }^{1} \cdot$ Thomas Hummel $^{1}$
}

Received: 10 October 2018 / Accepted: 10 December 2018 / Published online: 15 December 2018

(c) The Author(s) 2018

\begin{abstract}
Purpose To provide up-to-date and detailed normative data based on a large-scale sample, increasing diagnostic validity by reference to narrow age groups as previous normative values were based upon smaller sample sizes-especially in the group of older subjects.

Method Data were obtained from 9139 healthy subjects (4928 females aged 5-96 years and 4211 males aged 5-91 years). The standard "Sniffin' Sticks" test was applied, comprising threshold (T), discrimination (D) and identification (I) subtests, and yielding a TDI sum score.

Results Hyposmia was established at a TDI score of less than 30.75. Age-related changes were found in each domain, most pronounced for thresholds. Individuals aged 20-30 years performed best, whereas children below the age of 10 and adults above the age of 71 scored only half as well. Sex-related differences were in favor of women.

Conclusions Data provide guidance for assessing individual olfactory performance in relation to specific age groups. Significant gender and age effects were observed, with a most pronounced increase of olfactory test scores between age 5 through 20 years and a dramatic decrease at the age of 60 through 71 years.
\end{abstract}

Keywords Olfaction · Sniffin' Sticks · Threshold · Discrimination · Identification · Normative data

\section{Introduction}

The "Sniffin' Sticks" test is a widely used tool for assessment of olfactory performance consisting of three subtests: olfactory threshold, odor discrimination and odor identification. It has been introduced over 20 years ago by Kobal et al. [1]. Since the first publication, test-retest reliability and validity have been established [2,3] and the test has

Thomas Hummel

thummel@mail.zih.tu-dresden.de

1 Department of Otorhinolaryngology, Smell and Taste Clinic, Technische Universität Dresden, Fetscherstraße 74, 01307 Dresden, Germany

2 Institute of Psychology, University of Wroclaw, ul. Dawida 1, 50527 Wrocław, Poland

3 Department of Neuropediatric Medicine, Technische Universität Dresden, Fetscherstraße 74, 01307 Dresden, Germany

4 Department of Psychotherapy and Psychosomatic Medicine, Technische Universität Dresden, Fetscherstraße 74, 01307 Dresden, Germany been successfully adapted across cultures, e.g., [4-6]. Both extended $[7,8]$ and abridged versions, with satisfying psychometric properties [9-11], have been proposed, along with modifications of the set of odors utilized [12-14].

The "Sniffin' Sticks" battery is used in daily clinical practice as well as scientific research. Individual scores can be related to standard values for (a) normosmia (normal olfactory function), (b) hyposmia (impaired olfactory function) or (c) functional anosmia (residual or absent olfactory function). Additionally, there is the category of supersmellers, i.e., subjects with an extraordinary sense of smell. Although norms for the Sniffin' Sticks test have already been published [15, 16], an update appeared advisable, based upon a large-scale sample comprising detailed age groups, as older subjects were underrepresented in previous studies. Furthermore, updated norms are necessary to monitor potential changes in olfactory performance caused by macro-scale environmental and social factors, e.g., pollution or dietary habits.

Here we present updated normative data for clinical and scientific quantitative assessment of olfactory performance in female and male subjects, with a threefold number of 
participants compared to previous studies. This large sample allowed us to bin individual results into age groups of 10 years each, for a more accurate reference of subjects' olfactory performance to their coevals. In addition, the narrow age categories resulted in more homogeneous groups and facilitated-although cross-sectional in nature-a detailed insight into the dynamics of olfactory performance during the course of life.

\section{Materials and methods}

Data were obtained from 9139 subjects [ 4928 females aged 5-96 years $(M=31.8, \mathrm{SD}=18.9)$ and 4211 males aged 5-91 years $(M=30.7, \mathrm{SD}=17.7)]$. Among them, 3432 (37.5\%) had been included in a previous study to establish normative data [15]. According to the inclusion criteria for the respective studies, all subjects were healthy and none reported histories for any olfactory disturbances.

Odors were delivered using felt-tip pens ("Sniffin' Sticks") of approximately $14 \mathrm{~cm}$ length and an inner diameter of $1.3 \mathrm{~cm}$. These pens carry a tampon soaked with $4 \mathrm{ml}$ of liquid odorant. For odor presentation, the cap was removed from the pen for approximately $3 \mathrm{~s}$, the pen's tip brought in front of the subject's nose and carefully moved from left to right nostril and backwards [3].

The threshold was obtained in a three alternative forced choice paradigm ( $3 \mathrm{AFC}$ ) where subjects were repeatedly presented with triplets of pens and had to discriminate one pen containing an odorous solution from two blanks filled with the solvent. Phenylethanol (dissolved in propylene glycol) or $n$-butanol (dissolved in water) were used, with both odorants having been found equivalent in olfactory sensitivity testing: scores obtained with both are correlated [17]. The highest concentration was a $4 \%$ odor solution. Sixteen concentrations were created by stepwise diluting previous ones by $1: 2$. Starting with the lowest odor concentration, a staircase paradigm was used where two subsequent correct identifications of the odorous pen or one incorrect answer marked a so-called turning point, and resulted in a decrease or increase, respectively, of concentration in the next triplet. Triplets were presented at $20 \mathrm{~s}$ intervals. The threshold score was the mean of the last four turning points in the staircase, with the final score ranging between 1 and 16 points.

The discrimination task used the same 3 AFC logic. Two pens of any triplet contained the same odorant, while the third pen smelled differently. Subjects were asked to indicate the single pen with a different smell. Within-triplet intervals were approximately $3 \mathrm{~s}$. As the odors used in this subtest were more intense, between-triplets intervals were 20-30 s. The score was the sum of correctly identified odors. Hence, the scores in this task ranged from 0 to 16 points. Importantly, subjects were blindfolded for the threshold and discrimination tasks to avoid visual identification of target pens.

Odor identification comprised common and familiar odorants (recognized by at least $75 \%$ of the population). Subjects were presented with single pens and asked to identify and label the smell, using four alternative descriptors for each pen. Between-pen intervals were approximately 20-30 s. The total score was the sum of correctly identified pens, thus subjects could score between 0 and 16 points.

The final "TDI score" was the sum of scores for Threshold, Discrimination and Identification subtests, with a range between 1 and 48 points.

\section{Statistical analyses}

Data were analyzed by means of SPSS v. 25 software (SPSS Inc., Chicago, Ill., USA). Subjects were divided into nine age groups: (A) 5-10 years $(n=889)$; (B) $11-20$ years $(n=1750)$; (C) 21-30 years $(n=2995)$; (D) $31-40$ years $(n=1102)$; (E) $41-50$ years $(n=847)$; (F) $51-60$ years $(n=737)$; $(\mathrm{G}) 61-70$ years $(n=464)$; $(\mathrm{H}) 71-80$ years $(n=212)$; and (I) over 81 years $(n=143)$. Descriptive statistics were computed to establish norms based on the extended sample (Table 1). We examined the effects of sex (female vs male) and age (groups A-I) on TDI scores by means of analysis of variance (ANOVA). Further, we modelled effects of sex and age on separate subtest scores obtained for threshold, discrimination and identification scores, controlling for within-subject variance using repeated measures analysis of variance (rm-ANOVA). Pairwise comparisons were Bonferroni-corrected for multiple comparisons between the nine age groups. To provide guidance for assessing individual olfactory abilities in relation to specific age groups, we calculated the tenth percentile of TDI score for each age group.

\section{Results}

\section{Effects of sex and age on overall TDI score}

We found a main effect of age on the overall TDI score $F(8$, $3337)=128.8, p<0.001, \eta^{2}=0.24$. Pairwise comparisons indicated that the most pronounced increase in overall olfactory abilities occurred between group A (5-10 years) and group B (11-20 years) and the most pronounced decrease at the age of 61-70 years (Figs. 1,2). There was also a significant yet small main effect of sex $F(1,3337)=26.9$, $p<0.001, \eta^{2}=0.008$, suggesting that on average females $(M=31.7 \pm 0.18)$ outperformed males $(M=30.4 \pm 0.19)$. The two factors of interest (sex and age) did not interact with each other $(p=0.12)$. 
Table 1 Normative values for the Sniffin' Sticks test

\begin{tabular}{|c|c|c|c|c|c|c|c|c|c|c|c|c|}
\hline & \multicolumn{4}{|c|}{ Female subjects } & \multicolumn{4}{|c|}{ Male subjects } & \multicolumn{4}{|c|}{ All subjects } \\
\hline & THR & DIS & ID & TDI & THR & DIS & ID & TDI & THR & DIS & ID & TDI \\
\hline \multicolumn{13}{|c|}{ Age group A: $5-10$ years } \\
\hline$N$ & 138 & 76 & 340 & 21 & 170 & 93 & 314 & 33 & 308 & 169 & 654 & 54 \\
\hline Mean & 7.59 & 10.83 & 12.16 & 27.13 & 6.95 & 10.35 & 12.10 & 23.99 & 7.24 & 10.57 & 12.13 & 25.21 \\
\hline SD & 3.01 & 1.94 & 2.46 & 4.95 & 3.29 & 2.29 & 2.39 & 3.60 & 3.18 & 2.15 & 2.43 & 4.41 \\
\hline Minimum & 1 & 6 & 2 & 18.25 & 1 & 6 & 4 & 18.5 & 1 & 6 & 2 & 18.25 \\
\hline Maximum & 16 & 16 & 16 & 35.75 & 15.5 & 16 & 16 & 31.75 & 16 & 16 & 16 & 35.75 \\
\hline \multicolumn{13}{|l|}{ Percentiles } \\
\hline 5 & 3.25 & 8 & 7.05 & 18.35 & 2.28 & 7 & 8 & 19.2 & 2.75 & 7 & 8 & 19.06 \\
\hline 10 & 3.95 & 8 & 9 & 19.25 & 3 & 8 & 9 & 19.5 & 3.25 & 8 & 9 & 19.38 \\
\hline 25 & 5.19 & 9 & 11 & 24.5 & 4.25 & 8.5 & 10 & 20.13 & 5 & 9 & 11 & 21 \\
\hline 50 & 7.5 & 11 & 12 & 28 & 6.75 & 10 & 12 & 23.75 & 7.125 & 11 & 12 & 25 \\
\hline 75 & 9.25 & 12 & 14 & 30 & 9 & 12 & 14 & 26.5 & 9 & 12 & 14 & 28.81 \\
\hline 90 & 11.53 & 13 & 15 & 34.6 & 11.75 & 13 & 15 & 29.45 & 11.75 & 13 & 15 & 30.63 \\
\hline 95 & 13.05 & 14.15 & 16 & 35.73 & 13.11 & 14 & 16 & 31.23 & 13 & 14 & 16 & 32.69 \\
\hline \multicolumn{13}{|c|}{ Age group B: 11-20 years } \\
\hline$N$ & 439 & 316 & 759 & 229 & 363 & 231 & 645 & 155 & 802 & 547 & 1405 & 384 \\
\hline Mean & 8.69 & 12.86 & 12.98 & 34.53 & 8.32 & 12.46 & 12.86 & 33.20 & 8.52 & 12.69 & 12.92 & 34.00 \\
\hline $\mathrm{SD}$ & 2.61 & 1.83 & 1.87 & 4.03 & 2.86 & 1.96 & 1.81 & 4.25 & 2.73 & 1.90 & 1.84 & 4.17 \\
\hline Minimum & 1 & 8 & 6 & 24 & 1 & 6 & 4 & 20.75 & 1 & 6 & 4 & 20.75 \\
\hline Maximum & 16 & 16 & 16 & 44.5 & 16 & 16 & 16 & 45 & 16 & 16 & 16 & 45 \\
\hline \multicolumn{13}{|l|}{ Percentiles } \\
\hline 5 & 4.75 & 10 & 10 & 27.13 & 3.5 & 9 & 9 & 26.1 & 4.277 & 9 & 10 & 26.56 \\
\hline 10 & 5.75 & 10 & 10 & 29.5 & 4.75 & 10 & 10.6 & 27.75 & 5.5 & 10 & 10 & 28.5 \\
\hline 25 & 7 & 12 & 12 & 32.25 & 6.5 & 11 & 12 & 30.75 & 6.75 & 12 & 12 & 31.5 \\
\hline 50 & 8.5 & 13 & 13 & 34.5 & 8 & 13 & 13 & 32.75 & 8.25 & 13 & 13 & 34 \\
\hline 75 & 10.25 & 14 & 14 & 37.25 & 10.25 & 14 & 14 & 36.5 & 10.25 & 14 & 14 & 36.75 \\
\hline 90 & 12 & 15 & 15 & 39.75 & 12.12 & 15 & 15 & 39.25 & 12 & 15 & 15 & 39.25 \\
\hline 95 & 13.75 & 16 & 16 & 41.25 & 13.25 & 15 & 15 & 39.6 & 13.5 & 15 & 16 & 40.5 \\
\hline \multicolumn{13}{|c|}{ Age group C: $21-30$ years } \\
\hline$N$ & 857 & 741 & 1523 & 716 & 649 & 600 & 1310 & 576 & 1506 & 1341 & 2833 & 1292 \\
\hline Mean & 9.35 & 13.17 & 13.61 & 36.23 & 9.11 & 12.89 & 13.63 & 35.70 & 9.25 & 13.04 & 13.62 & 35.99 \\
\hline SD & 3.00 & 1.84 & 1.97 & 4.07 & 2.96 & 1.88 & 1.72 & 4.35 & 2.98 & 1.86 & 1.86 & 4.20 \\
\hline Minimum & 1 & 5 & 0 & 23 & 2.5 & 5 & 5 & 18 & 1 & 5 & 0 & 18 \\
\hline Maximum & 16 & 16 & 16 & 48 & 16 & 16 & 16 & 47 & 16 & 16 & 16 & 48 \\
\hline \multicolumn{13}{|l|}{ Percentiles } \\
\hline 5 & 5 & 10 & 9.75 & 29.96 & 4.75 & 10 & 11 & 29 & 5 & 10 & 10 & 29.5 \\
\hline 10 & 5.75 & 11 & 11 & 31 & 5.5 & 11 & 11 & 30.25 & 5.75 & 11 & 11 & 30.75 \\
\hline 25 & 7.5 & 12 & 13 & 33.5 & 7 & 12 & 13 & 32.75 & 7.25 & 12 & 13 & 33.06 \\
\hline 50 & 8.75 & 13 & 14 & 36 & 8.5 & 13 & 14 & 35.5 & 8.5 & 13 & 14 & 35.75 \\
\hline 75 & 11.25 & 14 & 15 & 38.75 & 11 & 14 & 15 & 38.5 & 11.25 & 14 & 15 & 38.5 \\
\hline 90 & 14 & 15 & 16 & 41.5 & 13.5 & 15 & 16 & 41.5 & 14 & 15 & 16 & 41.5 \\
\hline 95 & 15 & 16 & 16 & 43.5 & 14.9 & 15 & 16 & 43 & 15 & 16 & 16 & 43.09 \\
\hline \multicolumn{13}{|c|}{ Age group D: $31-40$ years } \\
\hline$N$ & 282 & 273 & 539 & 270 & 216 & 211 & 542 & 208 & 498 & 484 & 1081 & 478 \\
\hline Mean & 9.14 & 12.93 & 13.64 & 35.94 & 8.66 & 12.67 & 13.63 & 35.05 & 8.93 & 12.82 & 13.63 & 35.55 \\
\hline SD & 2.86 & 1.83 & 1.70 & 3.93 & 2.87 & 1.83 & 1.60 & 4.12 & 2.87 & 1.83 & 1.65 & 4.03 \\
\hline Minimum & 1 & 6 & 2 & 23.5 & 1.75 & 6 & 5 & 22.25 & 1 & 6 & 2 & 22.25 \\
\hline Maximum & 16 & 16 & 16 & 45.75 & 16 & 16 & 16 & 46 & 16 & 16 & 16 & 46 \\
\hline
\end{tabular}


Table 1 (continued)

\begin{tabular}{|c|c|c|c|c|c|c|c|c|c|c|c|c|}
\hline & \multicolumn{4}{|c|}{ Female subjects } & \multicolumn{4}{|c|}{ Male subjects } & \multicolumn{4}{|c|}{ All subjects } \\
\hline & THR & DIS & ID & TDI & THR & DIS & ID & TDI & THR & DIS & ID & TDI \\
\hline \multicolumn{13}{|l|}{ Percentiles } \\
\hline 5 & 4.79 & 10 & 11 & 29.5 & 4.25 & 10 & 11 & 27.48 & 4.421 & 10 & 11 & 28.74 \\
\hline 10 & 5.75 & 11 & 12 & 31 & 4.75 & 10 & 12 & 29.25 & 5.5 & 10 & 12 & 30.5 \\
\hline 25 & 7.5 & 12 & 13 & 33.5 & 6.5 & 11 & 13 & 32.76 & 7 & 12 & 13 & 33 \\
\hline 50 & 9 & 13 & 14 & 36.25 & 8.5 & 13 & 14 & 35 & 8.675 & 13 & 14 & 35.5 \\
\hline 75 & 11 & 14 & 15 & 38.75 & 10.69 & 14 & 15 & 37.5 & 10.75 & 14 & 15 & 38.5 \\
\hline 90 & 12.5 & 15 & 15 & 40.48 & 12.15 & 15 & 15 & 40.5 & 12.5 & 15 & 15 & 40.5 \\
\hline 95 & 15 & 15.3 & 16 & 42.5 & 14.04 & 15 & 16 & 41.39 & 14.5125 & 15 & 16 & 42.01 \\
\hline \multicolumn{13}{|c|}{ Age group E: $41-50$ years } \\
\hline$N$ & 199 & 198 & 456 & 197 & 171 & 170 & 390 & 170 & 370 & 368 & 846 & 367 \\
\hline Mean & 8.57 & 12.49 & 13.35 & 34.68 & 8.21 & 12.05 & 13.25 & 33.34 & 8.41 & 12.29 & 13.30 & 34.06 \\
\hline SD & 2.61 & 1.87 & 1.71 & 4.03 & 3.15 & 2.21 & 1.85 & 5.36 & 2.87 & 2.04 & 1.78 & 4.73 \\
\hline Minimum & 2.25 & 7 & 2 & 22.5 & 1 & 5 & 6 & 15.5 & 1 & 5 & 2 & 15.5 \\
\hline Maximum & 16 & 16 & 16 & 44 & 16 & 16 & 16 & 44.25 & 16 & 16 & 16 & 44.25 \\
\hline \multicolumn{13}{|l|}{ Percentiles } \\
\hline 5 & 4.5 & 9 & 10 & 26.98 & 2.9 & 7.55 & 10 & 22.66 & 3.5 & 9 & 10 & 25.5 \\
\hline 10 & 5.5 & 10 & 11 & 28.7 & 4.1 & 9 & 11 & 26.5 & 5 & 9 & 11 & 28.15 \\
\hline 25 & 6.75 & 11 & 12 & 32.5 & 6.25 & 11 & 12 & 30.44 & 6.5 & 11 & 12 & 31.5 \\
\hline 50 & 8.5 & 13 & 14 & 35.25 & 8.5 & 12 & 14 & 34 & 8.5 & 13 & 14 & 34.75 \\
\hline 75 & 10.25 & 14 & 14 & 37.25 & 10 & 14 & 14 & 36.5 & 10 & 14 & 14 & 37 \\
\hline 90 & 11.5 & 15 & 15 & 39.75 & 12.45 & 14 & 15 & 39.25 & 12 & 15 & 15 & 39.5 \\
\hline 95 & 13.75 & 15 & 16 & 41.03 & 14.15 & 15 & 16 & 41.11 & 13.75 & 15 & 16 & 41 \\
\hline \multicolumn{13}{|c|}{ Age group F: 51-60 years } \\
\hline$N$ & 221 & 215 & 401 & 213 & 175 & 173 & 331 & 172 & 396 & 388 & 732 & 385 \\
\hline Mean & 7.93 & 12.34 & 13.11 & 33.64 & 7.21 & 11.78 & 12.85 & 31.87 & 7.61 & 12.09 & 12.99 & 32.85 \\
\hline SD & 2.96 & 1.70 & 1.77 & 4.11 & 3.08 & 2.12 & 2.05 & 5.16 & 3.03 & 1.92 & 1.90 & 4.69 \\
\hline Minimum & 2.5 & 7 & 4 & 24 & 1 & 5 & 4 & 15 & 1 & 5 & 4 & 15 \\
\hline Maximum & 16 & 16 & 16 & 45 & 16 & 16 & 16 & 44 & 16 & 16 & 16 & 45 \\
\hline \multicolumn{13}{|l|}{ Percentiles } \\
\hline 5 & 3.5 & 9 & 10 & 26.5 & 2.5 & 8 & 9 & 21.33 & 3 & 9 & 9 & 25.33 \\
\hline 10 & 4.29 & 10 & 11 & 28.5 & 3.5 & 9 & 10 & 26 & 4 & 10 & 11 & 27.25 \\
\hline 25 & 5.63 & 11 & 12 & 30.75 & 4.75 & 10 & 12 & 29.25 & 5.5 & 11 & 12 & 30.34 \\
\hline 50 & 7.5 & 12 & 13 & 33.5 & 7.25 & 12 & 13 & 32.38 & 7.5 & 12 & 13 & 33 \\
\hline 75 & 9.5 & 14 & 14 & 36.5 & 9 & 13 & 14 & 35.25 & 9.25 & 13 & 14 & 36.25 \\
\hline 90 & 11.95 & 14 & 15 & 39.4 & 11 & 14 & 15 & 37.75 & 11.5 & 14 & 15 & 38.5 \\
\hline 95 & 13.95 & 15 & 16 & 40.65 & 12.8 & 15 & 16 & 39.5 & 13.5 & 15 & 16 & 40.18 \\
\hline \multicolumn{13}{|c|}{ Age group G: 61-70 years } \\
\hline$N$ & 141 & 133 & 255 & 133 & 118 & 111 & 209 & 111 & 259 & 244 & 464 & 244 \\
\hline Mean & 7.28 & 11.72 & 12.27 & 31.37 & 7.01 & 11.34 & 12.20 & 31.12 & 7.16 & 11.55 & 12.24 & 31.26 \\
\hline SD & 2.80 & 1.92 & 2.24 & 4.39 & 3.08 & 2.31 & 2.55 & 5.23 & 2.93 & 2.11 & 2.38 & 4.78 \\
\hline Minimum & 1 & 6 & 2 & 18.75 & 1 & 4 & 2 & 12 & 1 & 4 & 2 & 12 \\
\hline Maximum & 16 & 16 & 16 & 43 & 16 & 16 & 16 & 44 & 16 & 16 & 16 & 44 \\
\hline \multicolumn{13}{|c|}{ Percentiles } \\
\hline 5 & 2.78 & 8 & 7.8 & 23.73 & 1.25 & 7 & 7 & 22 & 2.5 & 8 & 7.25 & 22.5 \\
\hline 10 & 3.5 & 9 & 10 & 25.5 & 2.5 & 8 & 9 & 24 & 3.5 & 9 & 10 & 24.88 \\
\hline 25 & 5.5 & 10.5 & 11 & 29.13 & 5.44 & 10 & 11 & 28.5 & 5.5 & 10 & 11 & 28.5 \\
\hline 50 & 7.25 & 12 & 13 & 31.75 & 6.5 & 12 & 13 & 31.5 & 7 & 12 & 13 & 31.63 \\
\hline 75 & 8.75 & 13 & 14 & 34.25 & 8.5 & 13 & 14 & 34.5 & 8.5 & 13 & 14 & 34.25 \\
\hline 90 & 10.5 & 14 & 15 & 36.5 & 11 & 14 & 15 & 37.35 & 10.5 & 14 & 15 & 36.5 \\
\hline
\end{tabular}


Table 1 (continued)

\begin{tabular}{|c|c|c|c|c|c|c|c|c|c|c|c|c|}
\hline & \multicolumn{4}{|c|}{ Female subjects } & \multicolumn{4}{|c|}{ Male subjects } & \multicolumn{4}{|c|}{ All subjects } \\
\hline & THR & DIS & ID & TDI & THR & DIS & ID & TDI & THR & DIS & ID & TDI \\
\hline 95 & 11.73 & 14.3 & 15 & 37.5 & 12.6 & 14.4 & 16 & 38.6 & 11.75 & 14 & 15 & 38.26 \\
\hline \multicolumn{13}{|c|}{ Age group H: 71-80 years } \\
\hline$N$ & 105 & 75 & 122 & 75 & 63 & 40 & 89 & 40 & 168 & 115 & 211 & 115 \\
\hline Mean & 5.68 & 10.65 & 11.20 & 28.44 & 5.06 & 10.20 & 10.71 & 26.96 & 5.45 & 10.50 & 10.99 & 27.93 \\
\hline SD & 2.73 & 2.73 & 2.77 & 5.71 & 3.06 & 2.47 & 2.94 & 6.87 & 2.86 & 2.64 & 2.85 & 6.15 \\
\hline Minimum & 1 & 4 & 3 & 11 & 1 & 5 & 3 & 9 & 1 & 4 & 3 & 9 \\
\hline Maximum & 13.5 & 16 & 16 & 41.25 & 12.5 & 15 & 16 & 38.5 & 13.5 & 16 & 16 & 41.25 \\
\hline \multicolumn{13}{|l|}{ Percentiles } \\
\hline 5 & 1.33 & 5.8 & 6 & 17.25 & 1 & 5.05 & 4 & 12.05 & 1 & 5.8 & 5 & 17.05 \\
\hline 10 & 2.5 & 6 & 7 & 19.2 & 1 & 6.1 & 7 & 16.6 & 1.5 & 6 & 7 & 19.2 \\
\hline 25 & 3.63 & 9 & 10 & 25.5 & 2.5 & 9 & 9 & 22.75 & 3.25 & 9 & 10 & 24.25 \\
\hline 50 & 5.5 & 11 & 11 & 28.5 & 4.5 & 10.5 & 11 & 27.38 & 5.25 & 11 & 11 & 28.5 \\
\hline 75 & 7.5 & 13 & 13 & 32.5 & 7.75 & 12 & 13 & 33.25 & 7.5 & 13 & 13 & 32.5 \\
\hline 90 & 9.5 & 14 & 14 & 35.5 & 9.05 & 13 & 14 & 34.25 & 9.275 & 14 & 14 & 34.9 \\
\hline 95 & 10.78 & 14.2 & 15 & 37.1 & 10.5 & 14.9 & 14 & 36.225 & 10.5 & 14.2 & 15 & 36.8 \\
\hline \multicolumn{13}{|c|}{ Age group I: over 81 years } \\
\hline$N$ & 106 & 19 & 107 & 20 & 33 & 16 & 36 & 16 & 139 & 35 & 143 & 36 \\
\hline Mean & 4.20 & 8.74 & 8.04 & 24.03 & 3.61 & 8.63 & 9.17 & 22.39 & 4.06 & 8.69 & 8.32 & 23.30 \\
\hline $\mathrm{SD}$ & 3.02 & 2.90 & 3.43 & 7.84 & 2.27 & 2.73 & 3.33 & 6.69 & 2.87 & 2.78 & 3.43 & 7.29 \\
\hline Minimum & 1 & 4 & 2 & 11.25 & 1 & 4 & 2 & 13 & 1 & 4 & 2 & 11.25 \\
\hline Maximum & 10.75 & 13 & 16 & 36.25 & 9 & 14 & 15 & 32.5 & 10.75 & 14 & 16 & 36.25 \\
\hline \multicolumn{13}{|c|}{ Percentiles } \\
\hline 5 & 1 & 4 & 2 & 11.29 & 1 & 4 & 4.55 & 13 & 1 & 4 & 2.2 & 11.89 \\
\hline 10 & 1 & 5 & 3.8 & 12.1 & 1 & 4.7 & 5 & 13.7 & 1 & 5 & 4 & 13 \\
\hline 25 & 1.25 & 6 & 6 & 17.06 & 1.125 & 7 & 6 & 15.56 & 1.25 & $\begin{array}{l}6 \\
9\end{array}$ & 6 & 16.69 \\
\hline 50 & 3.75 & 9 & 8 & 26.5 & 3.5 & 8.5 & 9.5 & 21.5 & 3.5 & & 8 & 25.75 \\
\hline 75 & 6.5 & 11 & 10 & 28.25 & 5.375 & 10 & 12 & 28.94 & 6.25 & 11 & 12 & 28.38 \\
\hline 90 & 8.65 & 13 & 13 & 35.98 & 6.9 & 12.6 & 13.3 & 31.45 & 8.5 & 12.4 & 13 & 32.8 \\
\hline 95 & 10.5 & & 14.6 & 36.25 & 7.6 & & 14.15 & & 10.25 & 13.2 & 14 & 36.25 \\
\hline
\end{tabular}

Results are listed separately for sex and the nine age groups (THR olfactory thresholds, DIS odor discrimination, ID odor identification, TDI composite score as the sum of results for threshold, discrimination, and identification)

\section{Effects of sex and age on olfactory threshold, odor discrimination and odor identification}

We observed a significant interaction between age group and subtest $\left[F(16,6646)=7.8, p<0.001, \eta^{2}=0.02\right]$, but not between sex and subtest $(p=0.23)$. The decrease was present in each test $\left(F=128.3, p<0.001, \eta^{2}=0.24\right)$; however, it was most pronounced in the threshold task as compared to discrimination and identification. Pairwise comparisons are displayed in Fig. 3.

With pooled genders, the tenth percentile of TDI score for group A (5-10 years) was 19.4 points; group B (11-20 years) 28.5 points; group C (21-30 years) 30.75 points; group D (31-40 years) 30.5 points; group E (41-50 years) 28.15 points; group F (51-60 years)
27.25 points; group $\mathrm{G}$ (61-70 years) 24.88 points; group H (71-80 years) 19.2 points; group I (over 81 years) 13 points. These data provide, on one hand, guidelines for assessing individual olfactory abilities in relation to specific age groups. On the other hand, the final diagnosis of anosmia versus normosmia depends on the reference group of young adults with a cutoff value of 30.75 points.

The term "functional anosmia" refers to individuals without any or with negligible-as experienced in everyday life-sense of smell. To differentiate between "functional anosmia" and hyposmia, we established a TDI score or 16 points, which is equivalent with both identification and discrimination scores of 8 , the maximum $90 \%$ of patients with anosmia would achieve, as reported earlier [16]. 
Fig. 1 Mean TDI scores obtained from female and male subjects across the nine age groups. Error bars represent SEM. The bottom table shows differences between mean scores of two groups (group in a column - group in a row) and the level of post-hoc test significance: $* * * p<0.001 ; * * p<0.01$ $* p<0.05$
Fig. 2 TDI scores obtained from female and male subjects with polynomial trendlines for both sexes

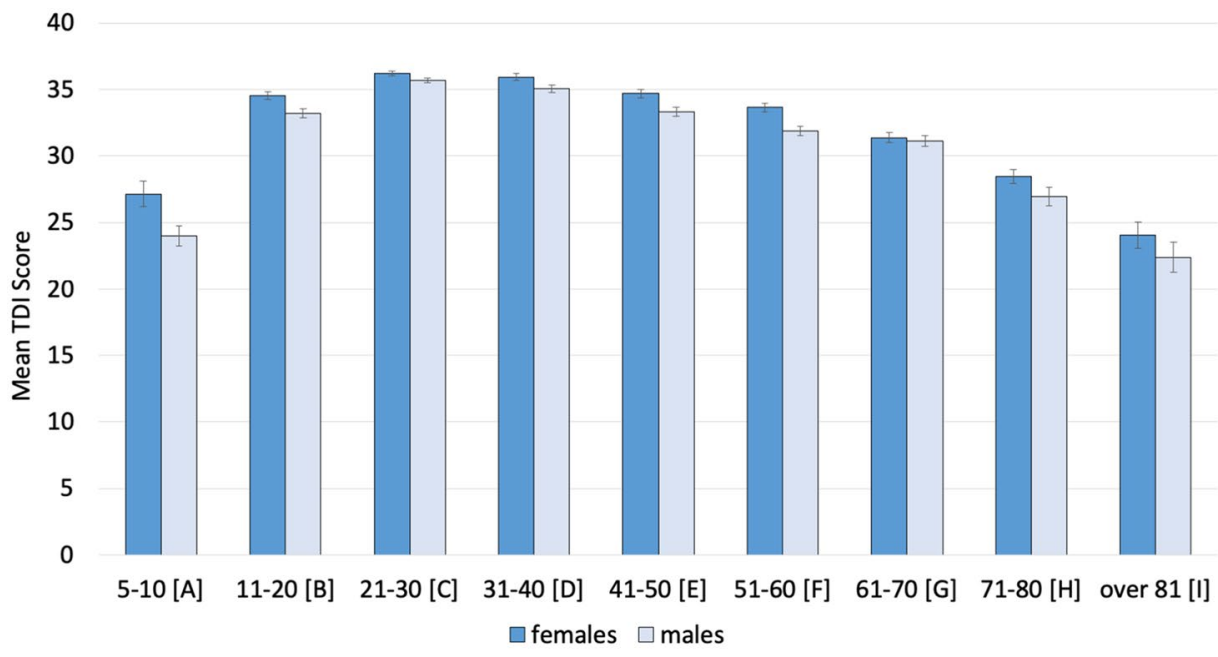

\begin{tabular}{|c|c|c|c|c|c|c|c|c|}
\hline $\begin{array}{l}\text { Age group } \\
\text { [Years] }\end{array}$ & $5-10$ & $11-20$ & $21-30$ & $31-40$ & $41-50$ & $51-60$ & $61-70$ & $71-80$ \\
\hline $11-20$ & $-8.31 * * *$ & & & & & & & \\
\hline $21-30$ & $-10.40 * * *$ & $-2.10^{* * *}$ & & & & & & \\
\hline $31-40$ & $-9.93 * * *$ & $-1.63^{* * *}$ & 0.47 & & & & & \\
\hline $41-50$ & $-8.45 * * *$ & -0.14 & $1.96 * * *$ & $1.49 * * *$ & & & & \\
\hline $51-60$ & $-7.19 * * *$ & $1.11^{*}$ & $3.21 * * *$ & $2.74 * * *$ & $1.26^{* *}$ & & & \\
\hline $61-70$ & $-5.68 * * *$ & $2.62^{* * *}$ & $4.72 * * *$ & $4.25 * * *$ & $2.76 * * *$ & $1.51^{* *}$ & & \\
\hline $71-80$ & -2.14 & $6.17 * * *$ & $8.26 * * *$ & $7.79 * * *$ & $6.31 * * *$ & $5.05 * * *$ & $3.54 * * *$ & \\
\hline over 81 & 2.35 & $10.66^{* * *}$ & $12.76 * * *$ & $12.29 * * *$ & $10.80 * * *$ & $9.55^{* * *}$ & $8.04 * * *$ & $4.50 * * *$ \\
\hline
\end{tabular}

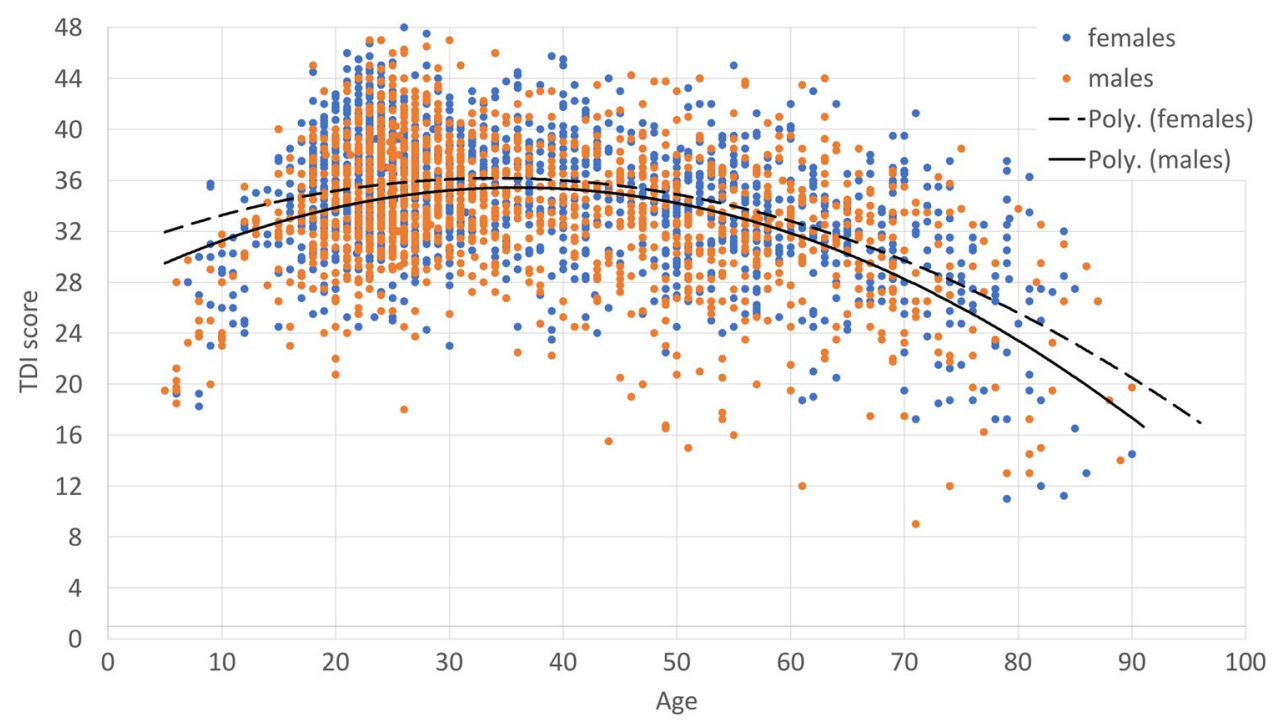

"Supersmellers" are subjects who reach at least the 90th percentile of the group aged 21-30 years, i.e., 41.5 or more points. Table 2 presents the proportion of subjects with functional anosmia (scoring $\leq 16$ points), hyposmia (scoring between 16.25 and 30.5 points), normosmia (scoring between 30.75 and 41.25 points), and supersmellers (scoring 41.5 points or above), across the nine age groups.

\section{Discussion}

The current study provides updated norms for the "Sniffin' Sticks" olfactory test based on a large sample. The present data obtained from 9139 subjects corroborate previous normative findings-which is noteworthy given that 
approximately two-thirds of the data are newly added to the database, as compared to the previous version from 2007 [15]. We observed similar values of the tenth percentile in all age groups, although the exact comparison cannot be made due to the more narrow age categories in the present study, e.g., previously, age group A was 5-15 years, whereas in the current study we present data for age groups A (5-10 years) and B (11-20 years).

With the current investigation we found the hyposmia cutoff point of 30.75 points in the reference group aged 21-30 years. Hitherto, the hyposmia cutoff score was 30.5 points [15] and in the proposed normative dataset, the same exact value of 30.5 points is the tenth percentile value of age group D (31-40 years). This 0.25 point difference between age groups $\mathrm{C}$ and $\mathrm{D}$ is likely to result from the division of the previously investigated group aged 16-35 years into two decade-wide age groups C (21-30 years) and D (31-40 years). The diagnosis of hyposmia remains to some extent an arbitrary decision, as the cutoff point of 30.75 has been established with respect to group C aged 21-30 years, representing the overall best smelling subsample. By a shift of perspective, individual scores may also be regarded in relation to the corresponding sex and age groups. We would like to give an example of how to interpret a patient's score: A female subject aged 55 years obtained a threshold score of 4.5 points, an identification of 14 points and a discrimination score of 13 points, resulting in a TDI score of 31.5 points. According to the tenth percentile of her age group, her outcome would be "hyposmia" for the threshold test and "normosmia" for identification and discrimination. As, by definition, the more general, overall TDI sumscore overrides separate subtest results, her final diagnosis would be "normosmia".

Importantly, changes of the tenth percentile TDI scores observed in the youngest and oldest age groups provoke the question about a deepened and updated analysis of the changes in olfactory performance [18]. Current data indicated the most pronounced loss in olfactory threshold, whereas olfactory discrimination and identification are, for one, tested with suprathreshold concentrations of odors and are, in addition, largely determined by individual experience and conscious cognitive processes which decrease at a slower pace over time. The pronounced decrease of odor thresholds with age supports the idea that it represents damage to the periphery of the olfactory system to a stronger degree than diminished odor identification and discrimination which are more strongly related to higher cognitive processes (for discussion see: [19-21]).

The relatively high percentage of children under 10 years with hyposmia is considered to be due to test difficulty rather than low olfactory function. Therefore, age-appropriate olfactory tests are necessary and have indeed been developed $[9,11,22]$.
Our extended data further corroborated earlier reports on decreased olfactory abilities in age groups over 55 years [ 18 , 23-27]. The apparent decrease in olfactory performance in seniors older than 60 raises the question about dynamics of olfactory loss with age.

"Functional anosmia"- a residual ability to perceive odors with limited usefulness in daily life-was found in a total of $0.45 \%$ of the subjects, and it was mostly prevalent in the oldest age groups, with the most visible decrease of function from age 70 years upwards. These subjects either have no olfactory function left at all or exhibit a modest ability to perceive, discriminate or identify odors insufficient for enjoyable experience of foods and drinks or the ability to detect environmental hazards such as gas, fire or food gone bad. However, age itself should not be considered a cause of olfactory loss but rather an accompanying factor of neurodegenerative diseases, drug side-effects, etc. [28, 29].

The incidence of $0.45 \%$ of participants with functional anosmia is low compared to epidemiological studies (e.g., [30]). One reason may be that the TDI score is used as the basis to establish the diagnosis of "functional anosmia". However, using an odor identification score below eight points to determine the fraction of this population returns the number of $3.4 \%$. It has to be considered that all subjects entering the study maintained to have a fully functional olfactory system. Yet, we found a meaningful proportion of subjects scoring in the range of hyposmia or functional anosmia, who seem either not to be aware of their olfactory dysfunction or not to be bothered by it. Finally, it must be kept in mind that the majority of the currently described population is young and healthy. Therefore no conclusions regarding epidemiology of olfactory loss in the general population can be made based on this work.

We observed sex-related differences with women outperforming men. Available empirical reports on this issue are inconclusive, with some studies pointing to a female advantage in olfactory tasks over males [15, 31, 32] but others failing to confirm this difference [33]; for review see: [34]. In our large sample, we observed the main effects of sex indicating that females obtained significantly higher scores than males-however, the difference in mean TDI scores calculated for both groups was rather small (1.3 points). In such a large sample size, even very small absolute differences become significant. In any case, the current study confirmed that sex-related differences are present but may be small; in other words, if sex-related differences are observed at all, it is typically women outperforming men.

We present updated norms for "Sniffin' Sticks" based on a large sample of 9139 subjects. With this extended sample we found hyposmia to be defined at less than 30.75 points of TDI score in the group aged 21-30 years. Observed effects of sex and age corroborate previous norms by showing a significant decrease of olfactory abilities with age with a 


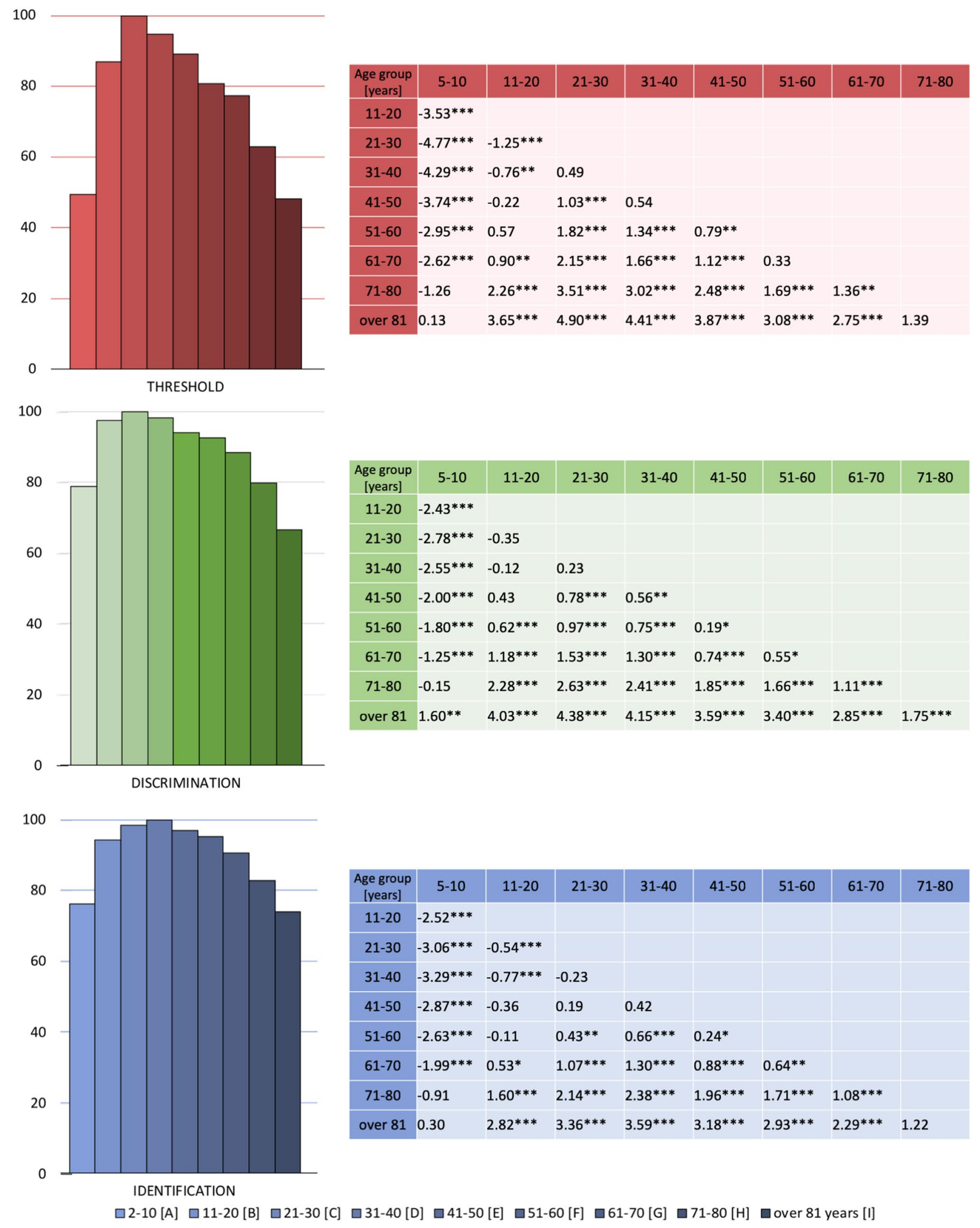


4 Fig. 3 Changes of odor threshold, discrimination, and identification across the nine age groups. All data were related to the respective average results obtained in reference to the age group with highest scores (group C for threshold and discrimination; group D for identification subtest). Tables in the right panel present differences between mean scores of two groups (group in a column-group in a row) and the level of post-hoc test significance: $* * * p<0.001 ; * * p<0.01$; $* p<0.05$

Table 2 Percentage of participants (all of whom identified themselves as having a normal sense of smell) with normosmia, hyposmia, and functional anosmia, separately for the nine age groups

\begin{tabular}{lllll}
\hline $\begin{array}{l}\text { Age group } \\
\text { (years) }\end{array}$ & $\begin{array}{l}\text { \% Func- } \\
\text { tional } \\
\text { anosmia }\end{array}$ & \% Hyposmia & \% Normosmia & $\begin{array}{l}\% \\
\text { Supers- } \\
\text { mellers }\end{array}$ \\
\hline $5-10$ & 0 & 90.7 & 9.3 & 0 \\
$11-20$ & 0 & 19.5 & 77.1 & 3.4 \\
$21-30$ & 0 & 9.6 & 79.4 & 11 \\
$31-40$ & 0 & 10.7 & 83.5 & 5.9 \\
$41-50$ & 0.3 & 20.7 & 75.2 & 3.8 \\
$51-60$ & 0.5 & 28.8 & 68.1 & 2.9 \\
$61-70$ & 0.4 & 38.5 & 59.4 & 1.6 \\
$71-80$ & 3.5 & 60 & 36.5 & 0 \\
Over 81 & 22.2 & 61.1 & 16.7 & 0 \\
\hline
\end{tabular}

most pronounced increase between age 5-20 years and a most pronounced decrease at the age of 60-71 years.

Acknowledgements We are indebted to all previous authors of the preceeding publications on this topic, especially Gerd Kobal, Hilmar Gudziol, and Alan MacKay-Sim. Authors would like to thank Cornelia Hummel for language editing and corrections.

Funding AO was supported by the Ministry of Science and Higher Education (\#626/STYP/12/2017). TH received a grant from the Deutsche Forschungsgemeinschaft (DFG HU 411/18-1).

\section{Compliance with ethical standards}

Conflict of interest Authors have no conflict of interest to disclose.

Ethical approval The study was performed in accordance with the Declaration of Helsinki on Biomedical Studies Involving Human Subjects.

Informed consent Informed written consent was obtained from all adult participants and children gave their assent to participate in a study. The data included were gathered from studies in healthy controls performed over the last 20 years, all of which have been approved individually by the local Ethics Review Board.

Open Access This article is distributed under the terms of the Creative Commons Attribution 4.0 International License (http://creativeco mmons.org/licenses/by/4.0/), which permits unrestricted use, distribution, and reproduction in any medium, provided you give appropriate credit to the original author(s) and the source, provide a link to the Creative Commons license, and indicate if changes were made.

\section{References}

1. Kobal G, Hummel T, Sekinger B et al (1996) "Sniffin Sticks": screening of olfactory performance. Rhinology 34:222-226

2. Haehner A, Mayer A-M, Landis BN et al (2009) High test-retest reliability of the extended version of the "Sniffin' Sticks". Test Chem Senses 34:705-711. https://doi.org/10.1093/chemse/bjp05 7

3. Hummel T, Sekinger B, Wolf SR et al (1997) 'Sniffin' Sticks': olfactory performance assessed by the combined testing of odour identification, odor discrimination and olfactory threshold. Chem Senses 22:39-52. https://doi.org/10.1093/chemse/22.1.39

4. Catana I, Negoiaș S, Maniu A et al (2014) A modified version of "Sniffin' Sticks" odor identification test: the romanian cultural adaptation. Clujul Med 85:218-223

5. Oleszkiewicz A, Taut M, Sorokowska A et al (2016) Development of the Arabic version of the "Sniffin' Sticks" odor identification test. Eur Arch Oto Rhino Laryngol. https://doi.org/10.1007/s0040 5-015-3718-2

6. Silveira-Moriyama L, Sirisena D, Gamage P et al (2009) Adapting the Sniffin' Sticks to diagnose Parkinson's disease in Sri Lanka. Mov Disord 24:1229-1233. https://doi.org/10.1002/mds.22545

7. Freiherr J, Gordon AR, Alden EC et al (2012) The 40-item monell extended Sniffin' Sticks identification test (MONEX-40). J Neurosci Methods 205:10-16. https://doi.org/10.1016/j.jneum eth.2011.12.004

8. Sorokowska A, Albrecht E, Haehner A, Hummel T (2015) Extended version of the "Sniffin" Sticks" identification test: testretest reliability and validity". J Neurosci Methods 243:111-114. https://doi.org/10.1016/j.jneumeth.2015.01.034

9. Gellrich J, Stetzler C, Oleszkiewicz A et al (2017) Olfactory threshold and odor discrimination ability in children-evaluation of a modified "sniffin' Sticks" test. Sci Rep. https://doi.org/10.1038/ s41598-017-01465-1

10. Hummel T, Rosenheim K, Konnerth C-G, Kobal G (2001) Screening of olfactory function with a four-minute odor identification test: reliability, normative data, and investigations in patients with olfactory loss. Ann Otol Rhinol Laryngol 110:976-981. https:// doi.org/10.1177/000348940111001015

11. Schriever VA, Mori E, Petters W et al (2014) The "Sniffin' Kids" test-a 14-Item odor identification test for children. PLoS One 9:e101086. https://doi.org/10.1371/journal.pone.0101086

12. Hsieh JW, Keller A, Wong M et al (2017) SMELL-S and SMELL$\mathrm{R}$ : olfactory tests not influenced by odor-specific insensitivity or prior olfactory experience. Proc Natl Acad Sci 114:11275-11284. https://doi.org/10.1073/pnas.1711415114

13. Oleszkiewicz A, Pellegrino R, Pusch K et al (2017) Chemical complexity of odors increases reliability of olfactory threshold testing. Sci Rep 7:39977. https://doi.org/10.1038/srep39977

14. Oleszkiewicz A, Würfel H, Han P, Hummel T (2018) Molecularly diverse odors advance olfactory threshold testing. J Sens Stud. https://doi.org/10.1111/joss.12440

15. Hummel T, Kobal G, Gudziol H, Mackay-Sim A (2007) Normative data for the "Sniffin'Sticks" including tests of odor identification, odor discrimination, and olfactory thresholds: an upgrade based on a group of more than. Eur Arch Oto Rhino Laryngol 264:237-243. https://doi.org/10.1007/s00405-006-0173-0

16. Kobal G, Klimek L, Wolfensberger M et al (2000) Multicenter investigation of 1036 subjects using a standardized method for the assessment of olfactory function combining tests of odor identification, odor discrimination, and olfactory thresholds. Eur Arch Oto Rhino Laryngol 257:205-211. https://doi.org/10.1007/s0040 50050223 
17. Croy I, Lange K, Krone F et al (2009) Comparison between odor thresholds for phenyl ethyl alcohol and butanol. Chem Senses 34:523-527. https://doi.org/10.1093/chemse/bjp029

18. Sorokowska A, Schriever VA, Gudziol V et al (2015) Changes of olfactory abilities in relation to age: odor identification in more than 1400 people aged 4 to 80 years. Eur Arch Oto Rhino Laryngol 272:1937-1944. https://doi.org/10.1007/s00405-014-3263-4

19. Hedner M, Larsson M, Arnold N et al (2010) Cognitive factors in odor detection, odor discrimination, and odor identification tasks. J Clin Exp Neuropsychol 32:1062-1067. https://doi. org/10.1080/13803391003683070

20. Whitcroft K, Cuevas M, Haehner A, Hummel T (2017) Patterns of olfactory impairment reflect underlying disease etiology. Laryngoscope 127:291-295. https://doi.org/10.1002/lary.26229

21. Hummel T, Whitcroft K, Andrews P et al (2017) Position paper on olfactory dysfunction. Rhinology 54:1-30. https://doi. org/10.4193/Rhin16.248

22. Schriever VA, Agosin E, Altundag A et al (2018) Development of an international odor identification test for children: the universal sniff test. J Pediatr 198:265-272.e3. https://doi.org/10.1016/J. JPEDS.2018.03.011

23. Hummel T, Futschik T, Frasnelli J, Hüttenbrink K-B (2003) Effects of olfactory function, age, and gender on trigeminally mediated sensations: a study based on the lateralization of chemosensory stimuli. Toxicol Lett 140:273-280. https://doi. org/10.1016/S0378-4274(03)00078-X

24. Konstantinidis I, Hummel T, Larsson M (2006) Identification of unpleasant odors is independent of age. Arch Clin Neuropsychol 21:615-621. https://doi.org/10.1016/j.acn.2006.05.006

25. Murphy C (1983) Age-related effects on the threshold, psychophysical function, and pleasantness of menthol. J Gerontol 38:217-222. https://doi.org/10.1093/geronj/38.2.217

26. Stuck BA, Frey S, Freiburg C et al (2006) Chemosensory eventrelated potentials in relation to side of stimulation, age, sex, and stimulus concentration. Clin Neurophysiol 117:1367-1375. https ://doi.org/10.1016/j.clinph.2006.03.004

27. Venstrom D, Amoore JE (1968) Olfactory threshold, in relation to age, sex or smoking. J Food Sci 33:264-265. https://doi. org/10.1111/j.1365-2621.1968.tb01364.x

28. Nordin S, Almkvist O, Berglund B (2012) Is loss in odor sensitivity inevitable to the aging individual? A study of "successfully aged" elderly. Chemosens Percept 5:188-196. https://doi. org/10.1007/s12078-011-9102-8

29. Mackay-Sim A, Johnston ANB, Owen C, Burne THJ (2006) Olfactory ability in the healthy population: reassessing presbyosmia. Chem Senses 31:763-771. https://doi.org/10.1093/chemse/ bj1019

30. Vennemann M, Hummel T, Berger K (2008) The association between smoking and smell and taste impairment in the general population. J Neurol 255:1121-1126. https://doi.org/10.1007/ s00415-008-0807-9

31. Doty RL, Applebaum S, Zusho H, Settle RG (1985) Sex differences in odor identification ability: a cross-cultural analysis. Neuropsychologia 23:667-672. https://doi.org/10.1016/00283932(85)90067-3

32. Doty RL, Cameron EL (2009) Sex differences and reproductive hormone influences on human odor perception. Physiol Behav 97:213-228. https://doi.org/10.1016/j.physbeh.2009.02.032

33. Sorokowska A, Hummel T (2014) Polish version of the Sniffin' Sticks Test-adaptation and normalization. Otolaryngol Pol 68:308-314. https://doi.org/10.1016/j.otpol.2014.08.001

34. Brand G, Millot J (2001) Sex differences in human olfaction: between evidence and enigma sex differences in human olfaction: between. Q J Exp Psychol Sect B 54:259-270. https://doi. org/10.1080/713932757 